Graphical abstract:

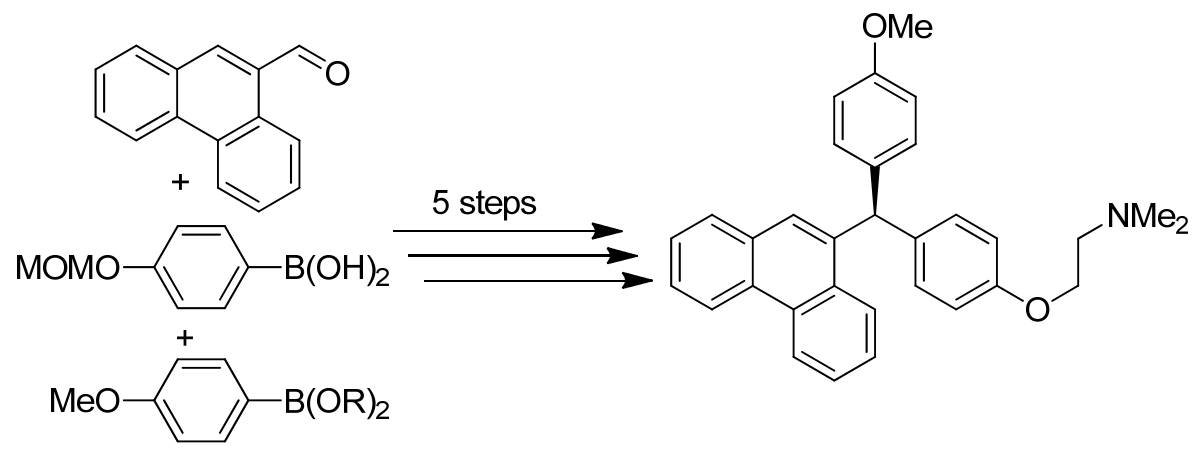




\title{
Selective synthesis of either enantiomer of an anti-breast cancer agent via a common enantioenriched intermediate
}

\author{
Aaron George Johnson, Marissa M. Tranquilli, Michael R. Harris, Elizabeth R. Jarvo* \\ Department of Chemistry, University of California, Irvine, Irvine, CA 92697 (USA) \\ http://chem.ps.uci.edu/ erjarvo/ \\ erjarvo@uci.edu
}

phone: (949) 824-7105; fax: (949) 824-8571

\begin{abstract}
:
A stereoselective synthesis of a bioactive triarylmethane is described. Key to the synthesis is a nickel-catalyzed Suzuki-Miyaura coupling which proceeds with retention at the benzylic center. This method is complementary to our previously reported nickelcatalyzed Kumada coupling which proceeds with inversion. Together, the two methods allow for efficient access to either enantiomer of biologically relevant triarylmethanes from a common enantioenriched intermediate.
\end{abstract}

Key words: nickel, cross-coupling, stereospecific, anti-cancer agent, triarylmethane

\section{Introduction}

Triarylmethanes are important targets due to their application in materials ${ }_{3}^{1}$ and medicinal chemistry (Figure 1).

In particular, the triarylmethane motif is found in anti-cancer lead compounds. ${ }^{3}$ One such compound is anti-breast cancer agent $1 .^{3 \mathrm{a}}$ In 2006, $( \pm)-\mathbf{1}$ was shown by Panda and co-workers to inhibit proliferation of the MCF-7 breast cancer cell line, with an in vitro $\mathrm{IC}_{50}$ of $3.88 \mu \mathrm{M}$. In vivo, $( \pm)-\mathbf{1}$ was shown to inhibit tumor growth and induce significant regression of mammary tumors in mice. Synthetic access to enantioenriched samples of triarylmethanes is critical for their evaluation as medicinal agents and in determining three-dimensional structure-activity relationships.
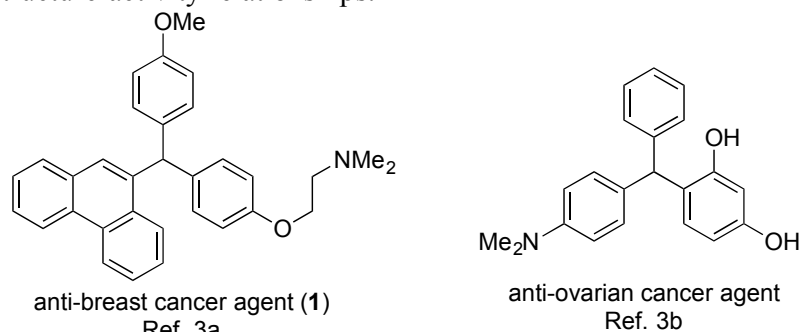

Ref. 3a

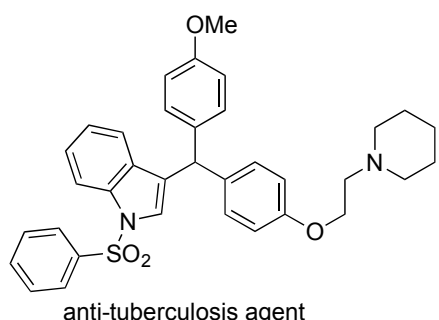

Ref. $4 a$ anti-ovarian cancer agent Ref. $3 b$

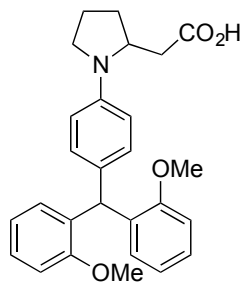

diabetes treatment Ref. 5
Figure 1. Bioactive triarylmethanes

Traditional syntheses of triarylmethanes, including $\mathbf{1}$, have relied on Friedel-Crafts reactions that lead to racemic products. $^{7,8}$ To broaden substrate scope beyond electron-rich aromatic compounds, access alternative regioisomers, and achieve stereocontrol, new methods have been developed. ${ }^{9,10}$ Recent advances include methods that utilize chiral Brønstead acids and $\mathrm{C}-\mathrm{H}$ bond activation. ${ }^{11,12,13}$ Approaches that develop
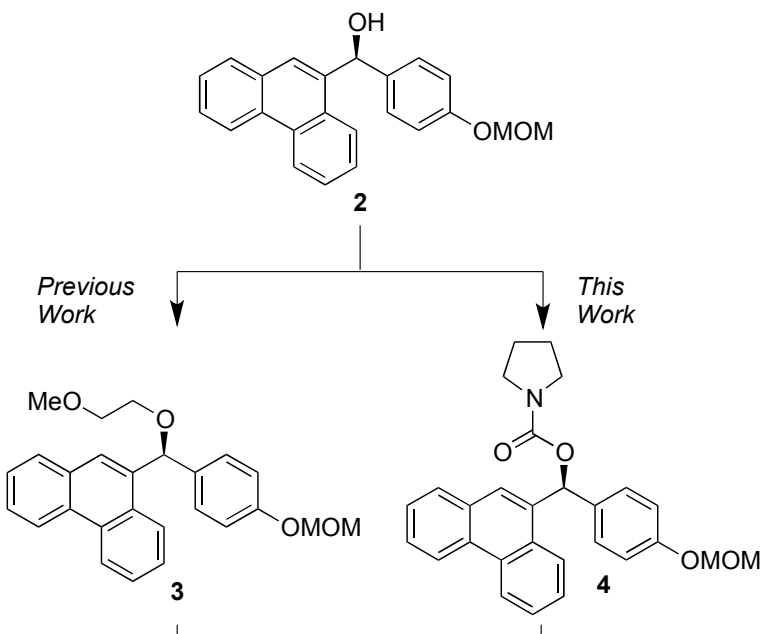

$\mathrm{Ni}(\operatorname{cod})_{2}(15 \mathrm{~mol} \%)$ dpph (30 mol \%) Kumada 4-MeOPhMgBr PhMe, RT, 72 h
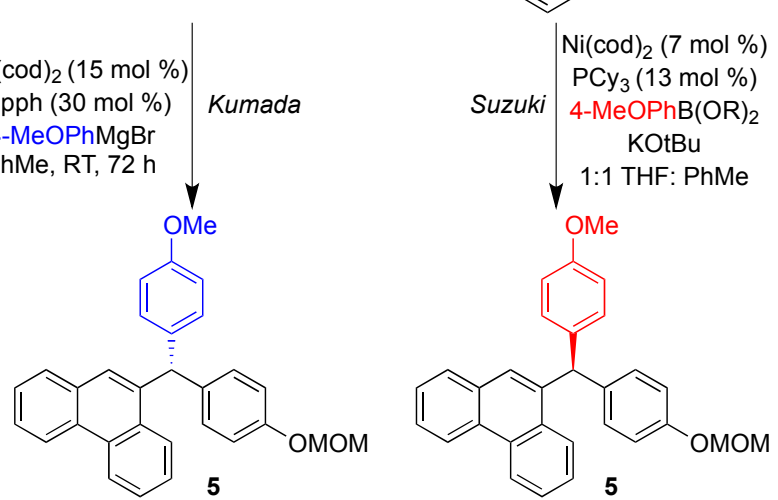

Scheme 1. Cross-coupling with inversion and retention

Suzuki and Kumada cross-coupling reactions have further increased access to enantioenriched triarylmethanes. Crudden and co-workers have demonstrated a palladium-catalyzed Suzuki reaction of enantioenriched boronic esters and aryl iodides. ${ }^{14}$ Our group has developed the umpolung approach in which benzylic ethers or esters are coupled with arylmetal reagents. ${ }^{15}$

Our contributions provide a strategy to prepare both enantiomers of a triarylmethane from a common enantiomer of 

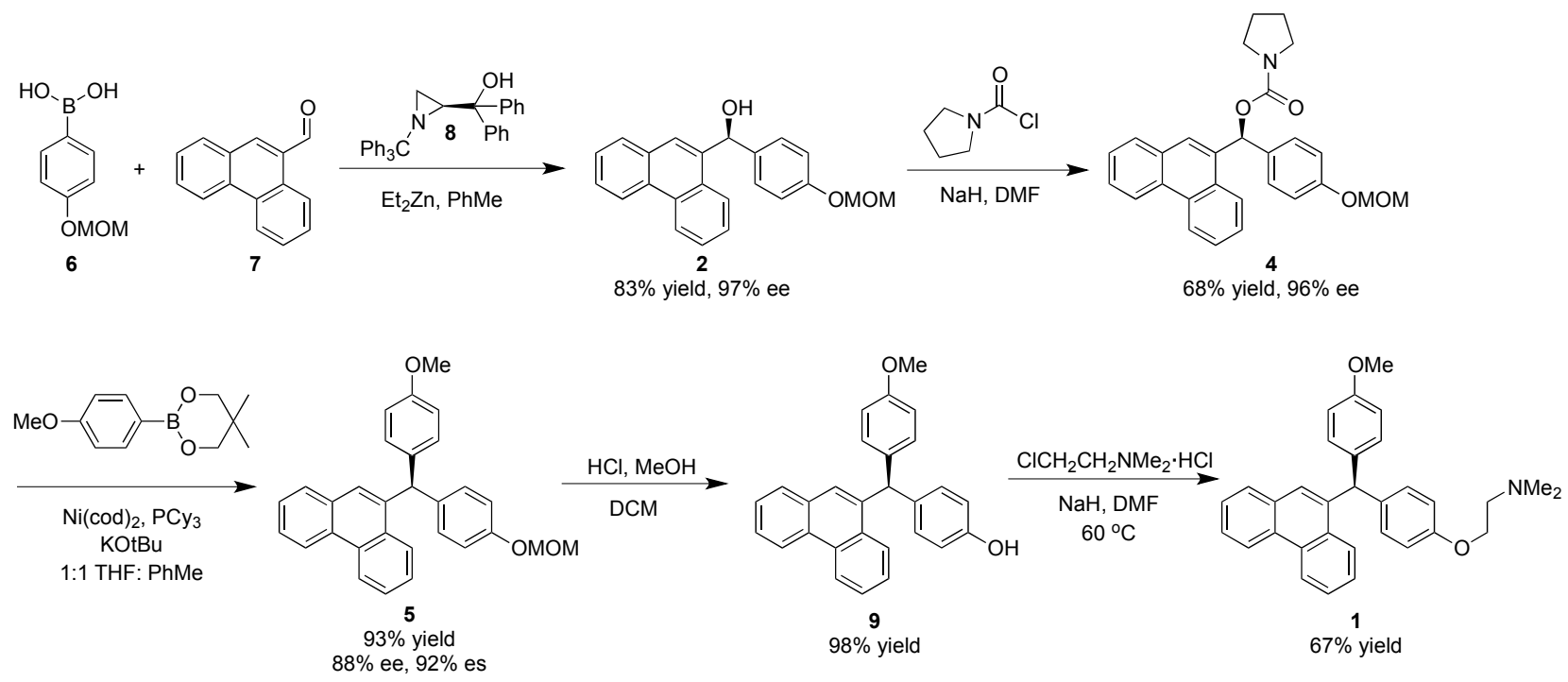

Scheme 2. Synthesis of 1 via Suzuki protocol

an alcohol intermediate. In 2012 we reported a stereospecific nickel-catalyzed Kumada cross-coupling reaction that allows for the preparation of optically active triarylmethanes from enantioenriched benzylic ethers such as 3 (Scheme 1). We employed this method in an enantioselective synthesis of $1 .{ }^{15 a}$ This Kumada reaction proceeds with inversion at the benzylic center. In 2013 our group reported a nickel-catalyzed stereospecific Suzuki-Miyaura reaction of enantioenriched diaryl carbamates, such as $\mathbf{4}$, with aryl boronic esters. ${ }^{15 b}$ In contrast to our Kumada protocol, the Suzuki reaction can proceed with retention of configuration at the benzylic center if tricyclohexyl phosphine is used as ligand, or inversion if SIMes is employed. Together, the Kumada and Suzuki reactions provide complementary methods to synthesize both enantiomers of $\mathbf{1}$ from the same enantiomer of alcohol 2. Herein we report the synthesis of the opposite enantiomer of $\mathbf{1}$ via the Suzuki reaction.

\section{Synthesis of 1}

The synthesis was carried out as described in Scheme 2. Enantioenriched alcohol $\mathbf{2}$ was prepared from commercially available boronic acid $\mathbf{6}$ and phenanthrene-9-carboxaldehyde 7 via an asymmetric arylation using chiral aziridine catalyst $\mathbf{8}$. 15a, 16 Alcohol $\mathbf{2}$ is the common intermediate for both the Kumada and Suzuki protocols. The Kumada protocol requires that the alcohol be converted into a methoxyethyl ether leaving group. The Suzuki reaction is more efficient if the alcohol is converted to an electron-withdrawing carbamate. The carbamate is installed by treatment of $\mathbf{2}$ with sodium hydride and 1-pyrrolidinecarbonyl chloride resulting in compound $\mathbf{4}$.

With carbamate 4 in hand, we were poised to test the key step, the stereospecific Suzuki cross-coupling reaction. Subjection of $\mathbf{4}$ to Suzuki conditions with tricyclohexylphosphine as ligand led to the efficient formation of triarylmethane 5 in $93 \%$ yield and $88 \%$ ee $\left(92 \%\right.$ es). ${ }^{17}$ Importantly, analysis of the reaction product by chiral SFC chromatography and comparison to material obtained by the Kumada route confirmed that the Suzuki reaction proceeds with overall retention. To achieve the highest yield, 4 was recrystallized prior to the cross-coupling reaction, as residual alcohol 2 diminishes the yield in the Suzuki coupling. Interestingly, when $\mathrm{PCy}_{3}$ is replaced with SIMes, compound 4 provides low yields of the desired Suzuki product. ${ }^{18}$

Completion of the synthesis was achieved by introduction of the requisite side chain. In the penultimate step of the synthesis the MOM group was removed under acidic conditions to form phenol 9. Finally, alkylation of the phenol by treatment with sodium hydride and 2-dimethylaminoethylchloride hydrochloride afforded target compound $1{ }^{19}$ This synthesis allowed for the preparation of $\mathbf{1}$ in $34 \%$ overall yield and $88 \%$ ee.

\section{Conclusion}

In summary, either enantiomer of anti-cancer triarylmethane 1 can be synthesized using a complementary pair of nickelcatalyzed cross-coupling reactions from a common enantiomer of a chiral alcohol intermediate. For the synthesis of anticancer agent 1, when the Kumada protocol is followed, the reaction occurs with inversion at the benzylic stereogenic center. When the Suzuki protocol is followed, the reaction occurs with retention at the benzylic stereogenic center, providing the opposite enantiomer of $\mathbf{1}$. These syntheses allow for efficient and selective preparation of either enantiomer of triarylmethanes for biological testing. With the exception of the alcohol protection and the subsequent nickel-catalyzed cross-coupling steps, both syntheses are identical and provide optically active $\mathbf{1}$ in five synthetic steps from commercially available starting materials. With both enantiomers in hand, our lab is carrying out studies on the potency of optically enriched $\mathbf{1}$ against a series cancer of cell lines.

\section{Acknowledgements}

This work was supported by NIH NIGMS (Ro1GM10o212), NSF-REU (CHE-1062891 CHEM-SURF fellowship to M.M.T.), and the National Science Foundation (Graduate Research Fellowship grant number DGE-1321846 to M.R.H.).

\section{References and Notes}


(1 ) (a) Duxbury, D. F. Chem. Rev. 1993, 93, 381. (b) Shchepinov, M. S.; Korshun, V. A. Chem. Soc. Rev. 2003, 32, 170. (c) Xu, Y.-Q.; Lu, J.-M.; Li, N.-J.; Yan, F.; Xia, X.-W.; Xu, Q.-F. Eur. Polym. J. 2008, 44, 2404. (d) Herron, N.; Johansson, G. A.; Radu, N. S.; US Patent Application 2005/0187364A1, Aug 25, 2005.

(2) For an overview of synthesis and medicinal properties of triarylmethanes, see: Mondal, F. S.; Panda, G. RSC Adv. 2014, 4, 28317.

(3) Anti-cancer: (a) Shagufta; Srivastava, A. K.; Sharma, R.; Mishra, R.; Balapure, A. K.; Murthy, P. S. R.; Panda, G. Bioorg. Med. Chem. 2006, 14, 1497. (b) Finer, J. T.; Chabala, J. C.; Lewis, E. US Patent Application 2004/0132830 A1, Jul 8, 2004. (c) Palchaudhuri, R.; Nesterenko, V.; Hergenrother, P. J. J. Am. Chem. Soc. 2008, 130, 10274.

(4) Anti-tuberculosis: (a) Panda, G.; Parai, M. K.; Das, S. K.; Shagufta; Manish, S.; Chaturvedi, V.; Srivastava, A. K.; Manju, Y. S.; Gaikwad, A. N.; Sinha, S. Eur. J. Med. Chem. 2007, 42, 410. (b) Parai, M. K.; Panda, G.; Chaturvedi, V.; Manju, Y. K.; Sinha, S. Bioorg. Med. Chem. Lett. 2008, 18, 289.

(5) Anti-diabetes: Ellsworth, B. A.; Ewing, W. R.; Jurica, E. U.S. Patent Application 2011/0082165 A1, Apr 7, 2011.

(6) Anti-implantation: Srivastava, N.; Sangita; Ray, S.; Singh, M. M.; Dwivedi, A.; Kumar, A. Bioorg. Med. Chem. 2004, 12, 1011.

(7) (a) Nair, V.; Thomas, S.; Mathew, S. C.; Abhilash, K. G.

Tetrahedron 2006, 62, 6731. (b) Pratt, E. F.; Green, L. Q. J. Am. Chem. Soc. 1953, 75, 275. (c) Muthyala, R; Katritzky, A. R.; Lan, X. Dyes Pigm. 1994, 25, 303.

(8) For more recent advances in transition-metal catalyzed Friedel-Crafts reactions: (a) Lin, S.; Lu, X. J. Org. Chem. 2007, 72, 9757. (b) Esquivias, J; Arrayás, R. G.; Carretero, J. C. Angew. Chem., Int. Ed. 2006, 45, 629. (c) Iovel, I.; Mertins, K.; Kischel, J.; Zapf, A.; Beller, M. Angew. Chem., Int. Ed. 2005, 44, 3913

(9) (a) Nambo, M.; Crudden, C. M. Angew. Chem., Int. Ed. 2014, 53, 742. (b) Xia, Y.; Hu, F.; Liu, Z.; Qu, P.; Ge, R.; Ma, C.; Zhang, Y.; Wang, J. Org. Lett. 2013, 15, 1784.

( 10 ) For cross-coupling strategies that provide racemic products, see: (a) Molander, G. A.; Elia, M. D. J. Org. Chem. 2006, 71, 9198. (b) Yu, J.-Y.; Kuwano, R. Org. Lett. 2008, 10, 973. (c) Tabuchi, S.; Hirano, K.; Satoh, T.; Miura, M. J. Org. Chem.

2014, 79, 5401 .

( 11 ) For chiral phosphoric acid catalyzed Friedel-Crafts alkylations, see: (a) Sun, F.-L.; Zheng, X.-J.; Gu, Q.; He, Q.-L., You, S.-L. Eur. J. Org. Chem. 2010, 47. (b) Zhuo, M.-H.; Jiang, Y.-J.; Fan, Y.-S.; Gao, Y.; Liu, S.; Zhang, S. Org. Lett. 2014, 16, 1096. (c) Saha, S.; Alamsetti, S. K.; Schneider, C. Chem. Commun. 2015, 51, 1461.

(12) For pyridine-directed enantioselective palladium-catalyzed C-H bond activation, see: (a) Shi, B.-F.; Maugel, N.; Zhang, Y.-H.; Yu, J.-Q. Angew. Chem., Int. Ed. 2008, 47, 4882.

(13) (a) Li, Y.-Z.; Li, B.-J.; Lu, X.-Y.; Lin, S.; Shi, Z.-J. Angew. Chem., Int. Ed. 2009, 48, 3817. (b) McGrew, G. I.; Temaismithi, J.; Carroll, P. J.; Walsh, P. J. Angew. Chem., Int. Ed. 2010, 49, 5541. (c) Zhang, J.; Bellomo, A.; Creamer, A. D.; Dreher, S. D.; Walsh, P. J. J. Am. Chem. Soc. 2012, 134, 13765. (d) Zhang, J.; Bellomo, A.; Trongsiriwat, N.; Jia, T.; Carroll, P. J.; Dreher, S. D.; Tudge, M. T.; Yin, H.; Robinson, J. R.; Schelter, E. J.;Walsh, P. J. J. Am. Chem. Soc. 2014, 136, 6276. (e) Niwa, T.; Yorimitsu, H.; Oshima, K. Org. Lett. 2007, 9, 2373.
(14) Matthew, S. C.; Glasspoole, B. W.; Eisenberger, P.; Crudden, C. M. J. Am. Chem. Soc. 2014, 136, 5828.

(15) (a) Taylor, B. L. H.; Harris, M. R.; Jarvo, E. R. Angew. Chem., Int. Ed. 2012, 51, 7790. (b) Harris, M. R.; Hanna, L. E.; Greene, M. A.; Moore, C. E.; Jarvo, E. R. J. Am. Chem. Soc. 2013, 135, 3303. (c) For a related transformation, see: Zhou, Q.; Srinivas, H. D.; Dasgupta, S.; Watson, M. P. J. Am. Chem. Soc. 2013, 135, 3307.

( 16 ) (a) Braga, A. L.; Paixão, M. W.; Westermann, B.; Schneider, P. H.; Wessjohann, L. A. J. Org. Chem. 2008, 73, 2879. (b) Bolm, C.; Rudolph, J. J. Am. Chem. Soc. 2002, 124, 14850.

(17) es $=$ enantiospecificity $=($ ee starting material $) /($ ee product $)$ see, Denmark, S. E.; Vogler, T. Chem. Eur. J. 2009, 15, 11737.

(18) Although effective with many benzhydryl carbamates, subjection of carbamate 4 to cross-coupling conditions using a catalyst prepared in situ from $\mathrm{Ni}(\mathrm{cod})_{2}$ and SIMes resulted in $10 \%$ yield of triarylmethane 5 . See reference $15 \mathrm{~b}$.

(19) McCague, R.; Leclercq, G.; Jordan, V. C. J. Med. Chem. 1988, 31,1285 . 